\title{
Patología dual en poblaciones especiales: una revisión narrativa
}

\author{
Néstor Szerman ${ }^{1,2}$, Rodrigo Marín-Navarrete³, José Fernández-Mondragón ${ }^{3}$, Carlos Roncero ${ }^{1,4}$ \\ 'Sociedad Española de Patología Dual (SEPD), España \\ ${ }^{2}$ Servicios de Salud Mental Retiro. Hospital Universitario Gregorio Marañón, Madrid, España \\ ${ }^{3}$ Unidad de Ensayos Clínicos en Adicciones y Salud Mental. Instituto Nacional de Psiquiatría Ramón de la Fuente Muñiz, \\ México \\ ${ }^{4}$ Sección de Adicciones y Patología Dual. Servicio de Psiquiatría. Hospital Universitario Vall d'Hebrón-ASPB, CIBERSAM. \\ Departamento de Psiquiatría Universidad Autónoma de Barcelona, España
}

\section{RESUMEN}

Introducción: la patología dual se refiere a la concurrencia de un trastorno por consumo de sustancias con otro trastorno psiquiátrico. Estudios epidemiológicos en población general reportan una alta prevalencia de patología dual. Sin embargo, la información disponible sobre el tema en poblaciones especiales es limitada. Objetivo: revisar la evidencia disponible sobre la epidemiología, la atención y los aspectos relevantes para la salud pública acerca de la patología dual en las siguientes poblaciones especiales: personas en situación de indigencia, en prisión, con VIH, VHB y VHC, mujeres y adultos mayores. Método: búsqueda en bases de datos bibliográficos (PubMed, Science Direct, EBSCO y OVID) de reportes de investigación enfocados en la prevalencia y el tratamiento de patología dual en las poblaciones mencionadas. Resultados: la prevalencia de la patología dual se presenta de manera diferenciada en estas poblaciones, en comparación con la población general; además está altamente asociada con mayores tasas de morbilidad y mortalidad. La mayoría de los estudios específicos sobre patología dual y las poblaciones especiales, tanto de epidemiología como de tratamientos, se ha realizado en países de alto ingreso. Discusión y conclusiones: se requiere de políticas públicas que contemplen la medición epidemiológica de la patología dual en estas poblaciones, sobre todo en países de mediano y bajo ingreso, e incluyan intervenciones integradas adecuadas a las necesidades específicas de cada población.

Palabras clave: patología dual; grupos minoritarios; epidemiología; revisión.

\begin{abstract}
Background: dual disorders is a term that refers to the co-occurrence of a substance use disorder with other mental disorders. Epidemiological studies have reported a high prevalence of dual disorders in the general population. However, information on dual disorders in special population is limited. Objective: to give an overview of the available evidence on the epidemiology, care and other relevant aspects for public health of dual disorders in the following special populations: homeless, prison inmates, people with sexually-transmitted infections, and the elderly. Method: search in peer-review publication databases (PubMed, Science Direct, EBSCO and OVID) for research reports on the epidemiology and treatment of dual pathology in the aforementioned populations. Results: prevalence of dual disorders in these populations appears to be different than in the general population; and it is highly associated with higher mortality and morbidity rates. The majority of studies aimed specifically on the epidemiology and treatment of dual disorders and special populations has been conducted in high-income countries. Discussion and conclusions: there is a need for public policies that consider the epidemiological assessment of dual disorders in special populations, specially in low-and middle-income countries, and include integrated care interventions adequate to the specific needs of each population.
\end{abstract}

Key words: dual disorders; minority groups; epidemiology; review.

\footnotetext{
Autor de correspondencia:

Néstor Szerman. Hospital Universitario Gregorio Marañón. C/ Lope de Rueda 43, 28009 Madrid, España. Tel.: +34 914008690

Fax: +34 91 5739932. Correo electrónico: nszerman@salud.madrid.org.

Recibido: 10 de julio del 2015.

Aceptado: 07 de septiembre del 2015.

DOI: 10.28931/riiad.2015.1.07
} 


\section{INTRODUCCIÓN}

La coexistencia de trastornos por consumo de sustancias (TCS) con otros trastornos psiquiátricos (OTP), también conocida como Patología Dual (PD) (Szerman et al., 2013), ha sido ampliamente documentada desde los años 80, con los estudios de epidemiología psiquiátrica de tercera generación (Borges, Medina-Mora, \& López-Moreno, 2004; Hasin \& Kilcoyne, 2012; Hasin, Nunes, \& Meylan, 2004; Kessler et al., 2004), que marcan un nuevo paradigma en la categorización de los trastornos mentales y sus comorbilidades. Aunque esto ha permitido avances en la comprensión de este fenómeno, los grandes estudios epidemiológicos en psiquiatría se basan en encuestas realizadas en hogares y población universitaria (Grant \& Dawson, 2006; Kessler et al., 2004; Demyttenaere et al., 2004), lo que limita el conocimiento sobre la salud mental en poblaciones marginadas (por ejemplo indigentes, reclusos, migrantes y refugiados) (Baxter, Patton, Scott, Degenhardt, \& Whiteford, 2013).

Según la Organización Mundial de la Salud (World Health Organization, 2010), el tratamiento y la prevención de los TCS a nivel global requieren estrategias que atiendan las necesidades específicas de las poblaciones especiales, definidas como grupos minoritarios que por alguna característica (género, orientación sexual, etnicidad, grupo etario, discapacidad, migración y marginación socioeconómica) están en desventaja con respecto a la población general, en cuanto a bienestar general y al otorgamiento de servicios de salud.

Por lo anterior, la presente revisión tiene como objetivo integrar la evidencia existente sobre epidemiología, etiología y atención de la PD en poblaciones especiales; haciendo énfasis en personas en situación de indigencia, en prisión, con VIH, VHB y VHC, mujeres y adultos mayores.

\section{MÉTODO}

Para realizar la presente revisión se consultaron libros de texto especializados, reportes epidemiológicos, reportes de investigación clínica y revisiones sistemáticas, publicados en revistas científicas indexadas. Para la integración de fuentes de consulta se realizó una búsqueda durante los meses de abril y mayo de 2015 en bases de datos (PubMed, ScienceDirect, EBSCO y OVID); se utilizaron los siguientes términos de búsqueda: "dual diagnosis", "substance misuse", "substance abuse", "mental disorders", "mental illness", "comorbidity", "co-occurring disorders", "gender differences", "women", "criminal justice", "inmates", "elderly", "older adults", "geriatric", "HIV/AIDS", "HCV", "HBV", "homeless", y "roofless", así como otros tér- minos específicos para indagar epidemiología, tratamiento y etiología de la PD en cada población especial.

Se consideraron para su inclusión, artículos escritos en inglés y en español. También se consultaron listas de referencias de artículos de revisión sistemática o de libros de texto, así como bases de datos en centros de información y documentación especializados en psiquiatría. Ante la necesidad de hacer una revisión comprehensiva de la evidencia sobre el tema, no se establecieron criterios de inclusión o exclusión a priori sobre los estudios y reportes de investigación consultados, sino que, durante la investigación, los autores proponían y discutían la inclusión de trabajos tomando en cuenta las siguientes características: publicaciones preferentemente de los últimos cinco años, valor pragmático (validez externa) y cumplimiento con criterios de calidad de los artículos; cabe mencionar que se buscaron los más apegados a criterios estándares internacionales (por ejemplo STROBE para estudios observacionales, CONSORT para ensayos clínicos, PRISMA para revisiones sistemáticas).

Una vez integradas todas las fuentes, se determinó organizar la evidencia encontrada en los siguientes apartados temáticos para cada una de las poblaciones especiales consideradas: a) aspectos epidemiológicos y b) implicaciones en salud pública (por ejemplo vulnerabilidad o exposición a riesgos de otras condiciones de salud, consideraciones para su tratamiento y prevención).

\section{RESULTADOS}

\section{Personas en situación de indigencia}

\section{Epidemiología}

Los TCS y los OTP juegan un papel importante en el estudio de la salud de las personas indigentes (personas que duermen y viven en situación de calle, en albergues, refugios $u$ hogares temporales). La indigencia es causada por una compleja interacción entre factores individuales (donde se incluyen de manera prominente los trastornos psiquiátricos y el consumo de sustancias) y factores socio/estructurales (por ejemplo aculturación, falta de oportunidades de educación, de una red social de apoyo y de opciones de alojamiento accesibles) que contribuyen a la exclusión social de la persona, limitan su acceso a tratamiento y exacerban la sintomatología psiquiátrica y adictiva (Fazel, Geddes, \& Kushel, 2014; Johnson \& Chamberlain, 2008; Montross et al., 2005). Se ha documentado que la coocurrencia de los TCS con OTP está fuertemente asociada con la prolongación y persistencia de la indigencia; además de estar vinculada con condiciones adversas durante la infancia, la adquisición de enfermedades infeccio- 
sas y el inicio a edad temprana de la indigencia (Patterson, Somers, \& Moniruzzman, 2012).

Diversos estudios han encontrado prevalencias de PD en esta población que pueden variar entre 10 y $60 \%$, siendo las concurrencias de TCS con trastornos psicóticos, afectivos y de ansiedad, las más prevalentes (Fazel, Khosla, Doll, \& Geddes, 2008; Fichter \& Quadflieg, 2005; Nielsen, Hjorthøj, Erlangsen, \& Nordentoft, 2011; Patterson et al., 2012; Torchalla, Strehlau, Li, \& Krausz, 2011). Con respecto a las diferencias de género, se ha encontrado que el riesgo de mortalidad atribuible a la PD es significativamente mayor en mujeres que en hombres (Nielsen et al., 2011). Un estudio realizado en mujeres reportó mayor prevalencia de dependencia de drogas (Torchalla et al., 2011) que de alcohol, lo que contrasta con lo hallado en estudios realizados en ambos géneros, donde la dependencia de alcohol es más prevalente (Montross et al., 2005). En la Tabla 1 se presenta un resumen de algunos estudios relevantes sobre patología dual en esta población.

Finalmente, se ha encontrado que las tasas de mortalidad en población indigente son más altas que en población general, lo cual puede atribuirse parcialmente a la presencia de trastornos psiquiátricos y al consumo de sustancias (Fazel et al., 2014). Se ha documentado que las principales causas de mortalidad en esta población están vinculadas con el uso de sustancias y los trastornos mentales, como los daños no-intencionados (caídas, quemaduras, accidentes de tránsito), las conductas autolesivas, los intentos de suicidio, el envenenamiento (por consumo de medicamentos o sustancias ilícitas) y los traumas cráneo-encefálicos (Beijer, Andreasson, Ågren, \& Fugelstad, 2011; Fazel et al., 2008; Nielsen et al., 2011; Pascual et al., 2008; Topolovec-Vranic et al., 2012; Unger et al., 1997).

Cabe mencionar que los estudios realizados sobre trastornos mentales en población indigente de países de mediano y bajo ingreso son limitados (Asante, Meyer-Weitz, \& Petersen, 2014; Konstantakopoulos et al., 2003; Shibre et al., 2015). Por ello, se ha hecho énfasis en la necesidad de basar las estrategias de atención a este grupo en esfuerzos locales de medición epidemiológica (Fazel et al., 2008; Fazel et al., 2014).

Implicaciones de salud pública

La PD puede afectar el curso clínico de las personas en situación de indigencia, pues se relaciona con un mayor número de recaídas, reingreso a hospitales, severidad de sintomatología, menor adherencia al tratamiento farmacológico, cambios marcados del estado de ánimo e ideación suicida, así como a una mayor exposición a violencia física y sexual, victimización, problemas le- gales (Jiménez-Castro, Raventós-Vorst, \& Escamilla, 2011; Kushel, Evans, Perry, Robertson, \& Moss, 2003) y un mayor riesgo de encarcelamiento (McNiel, Binder, \& Robinson, 2005). Asimismo, la indigencia está asociada con otras condiciones de salud como la desnutrición, la diabetes, el asma, las cardiopatías y las enfermedades infectocontagiosas (por ejemplo tuberculosis, hepatitis $\mathrm{B}$ [VHB], hepatitis C [VHC], VIH), en las que la presencia de la PD dificulta la provisión de atención adecuada (Fazel et al., 2008; Gonzáles \& Rosencheck, 2002).

Diversos estudios han reportado que las personas que mantienen contacto con los servicios de albergue $o$ utilizan en mayor medida los servicios de salud mental disponibles (particularmente durante los primeros meses de indigencia) presentan una mejora en su salud, tanto física como mental, así como una reducción en el tiempo que viven en situación de indigencia (Fichter \& Quadflieg, 2005; Hwang \& Burns, 2014; Schanzer, Domínguez, Shrout, \& Canton, 2007). A pesar de lo anterior, es mayor la proporción de servicios dirigidos a indigentes que excluyen a las personas con trastornos por consumo de sustancias, que la de aquéllos que cuentan con atención especializada para las adicciones (Canavan et al., 2012).

Por lo anterior, para el tratamiento de PD se ha hecho énfasis en el potencial de los enfoques integrales que buscan solucionar de manera conjunta tanto los problemas de salud como el de la indigencia (Hwang \& Burns, 2014; Polcin, 2015). Un ejemplo es el tratamiento asertivo comunitario ( $\mathrm{ACT}$ ), en el que además de proveer asistencia social (por ejemplo búsqueda de alojamiento estable y alimento), se proporcionan servicios de salud mental, en los que cada caso es atendido por equipos completos de profesionales (Nelson et al., 2014); en un ensayo clínico reciente, esta estrategia demostró efectividad en indigentes con trastornos mentales severos (Aubry et al., 2015), al compararla con la provisión por separado de atención para la salud y asistencia social. También se debate la pertinencia de los enfoques orientados a la reducción del daño (por ejemplo intervenciones que permiten a alcohólicos crónicos continuar bebiendo) que minimizan los costos de atención, contra las intervenciones orientadas a la abstinencia; así como el potencial del apoyo comunitario brindado por pares (Vet de et al., 2014; Hwang \& Burns, 2014; Hwang, Tolomiczenko, Kouyoumdjian, \& Garner, 2005; Polcin, 2015; Tsemberis, Gulcur, \& Nakae, 2004).

\section{Personas en prisión}

\section{Epidemiología}

La presencia de TCS y OTP es frecuente entre las personas que se encuentran en prisión. La literatura ha reportado que en la población penitenciaria existe una 
Tabla 1

Estudios observacionales sobre patología dual en personas en situación de indigencia

\begin{tabular}{|c|c|c|}
\hline Autores & Muestra y evaluación & Hallazgos \\
\hline \multirow[t]{4}{*}{$\begin{array}{l}\text { Fichter \& } \\
\text { Quadflieg (2005) }\end{array}$} & $\begin{array}{l}265 \text { hombres indigentes } \\
\text { seguidos a lo largo de tres } \\
\text { años, a través de agencias } \\
\text { de trabajo social, centros de } \\
\text { reunión y albergues. }\end{array}$ & $\begin{array}{l}\text { Los trastornos más prevalentes en la muestra fueron los TCS, con } \\
69.66 \% \text { basal y } 54.19 \% \text { en el seguimiento. }\end{array}$ \\
\hline & $\begin{array}{l}\text { Entrevista clínica estructurada } \\
\text { para el DSM-IV (SCID-I). }\end{array}$ & $\begin{array}{l}\text { Los trastornos psicóticos presentaron una prevalencia de } 4.38 \% \text { a } \\
\text { nivel basal y } 5.70 \% \text { en el seguimiento, mostrando la tasa más alta de } \\
\text { incidencia entre las comorbilidades psiquiátricas (1.38\%). }\end{array}$ \\
\hline & & $\begin{array}{l}\text { Los trastornos ansiosos presentaron una prevalencia de } 10.98 \% \text { con } \\
\text { una tasa de remisión de } 7.61 \% \text {; los trastornos del estado de ánimo } \\
\text { contaron con una prevalencia de } 19.17 \% \text { con una remisión de } 8.22 \% \text {. }\end{array}$ \\
\hline & & $\begin{array}{l}\text { El trastorno con mayor tasa de incidencia fue la dependencia al } \\
\text { policonsumo de sustancias, con } 2.28 \% \text {. }\end{array}$ \\
\hline \multirow[t]{3}{*}{$\begin{array}{l}\text { Montross et al. } \\
(2005)\end{array}$} & $\begin{array}{l}6,424 \text { registros de pacientes } \\
\text { bajo tratamiento para la } \\
\text { esquizofrenia o trastorno }\end{array}$ & $\begin{array}{l}\text { Un total de } 1,397 \text { pacientes registrados }(21.7 \%) \text { presentaron } \\
\text { concurrencia de un TCS. }\end{array}$ \\
\hline & esquizoafectivo. & $\begin{array}{l}\text { La indigencia y el género masculino, así como la aculturación } \\
\text { (marginación) fueron factores predictores de la comorbilidad. }\end{array}$ \\
\hline & $\begin{array}{l}\text { Diagnóstico confirmado por } \\
\text { psiquiatra. }\end{array}$ & \\
\hline \multirow[t]{3}{*}{$\begin{array}{l}\text { Nielsen et al. } \\
\text { (2011) }\end{array}$} & $\begin{array}{l}32,711 \text { registros médicos de } \\
\text { personas indigentes tomados } \\
\text { entre } 1999 \text { y } 2009 \text {. }\end{array}$ & $\begin{array}{l}62.4 \% \text { de los hombres y } 58.2 \% \text { de las mujeres presentaron cualquier } \\
\text { diagnóstico psiquiátrico. }\end{array}$ \\
\hline & $\begin{array}{l}\text { Criterios CIE-10 para trastornos } \\
\text { afectivos, esquizofrenia, } \\
\text { trastornos por consumo de } \\
\text { sustancias y trastornos de la }\end{array}$ & $\begin{array}{l}10.9 \% \text { de los hombres y } 7.3 \% \text { de las mujeres presentaron patología } \\
\text { dual (esquizofrenia y TCS) y un aumento en el riesgo de mortalidad } \\
\text { en ambos sexos } 1.3 \text { ( } 95 \% \text { IC 1.2-1.5) y } 1.7 \text { ( } 95 \% \text { IC 1.3-2.1), } \\
\text { respectivamente. }\end{array}$ \\
\hline & personalidad. & $\begin{array}{l}\text { La prevalencia de otros trastornos psiquiátricos en hombres y mujeres } \\
\text { (afectivos y de la personalidad) fue de } 10.1 \% \text { y } 17.1 \% \text {, respectivamente. }\end{array}$ \\
\hline \multirow[t]{3}{*}{$\begin{array}{l}\text { Patterson et al. } \\
\text { (2012) }\end{array}$} & $\begin{array}{l}425 \text { indigentes reclutados en } \\
\text { albergues, agencias de trabajo } \\
\text { social, hospitales, programas }\end{array}$ & $\begin{array}{l}52 \% \text { de la muestra presentó de manera simultánea más de un trastorno } \\
\text { mental. }\end{array}$ \\
\hline & $\begin{array}{l}\text { de justicia criminal y servicios } \\
\text { comunitarios de salud mental. }\end{array}$ & $\begin{array}{l}\text { La patología dual fue significativamente más prevalente en la muestra } \\
\text { con mayor tiempo de indigencia (>3 años) (59\%), así como la } \\
\text { dependencia de sustancias ( } 67 \%) \text {. }\end{array}$ \\
\hline & $\begin{array}{l}\text { Mini entrevista } \\
\text { neuropsiquiátrica internacional } \\
(\mathrm{MINI}) \text {. }\end{array}$ & $\begin{array}{l}\text { Los trastornos más prevalentes en la muestra fueron la dependencia de } \\
\text { sustancias }(58 \%) \text {, los trastornos psicóticos }(52 \%) \text { y la depresión mayor } \\
(40 \%) \text {, }\end{array}$ \\
\hline \multirow[t]{3}{*}{$\begin{array}{l}\text { Torchalla et al. } \\
\text { (2011) }\end{array}$} & $\begin{array}{l}196 \text { mujeres indigentes } \\
\text { reclutadas en la calle. }\end{array}$ & $\begin{array}{l}58.3 \% \text { de la muestra presentó cualquier trastorno mental de manera } \\
\text { concurrente con uso de sustancias. }\end{array}$ \\
\hline & $\begin{array}{l}\text { Mini entrevista } \\
\text { neuropsiquiátrica internacional } \\
(\mathrm{MINI}) \text {. }\end{array}$ & $\begin{array}{l}\text { La dependencia de drogas presentó una prevalencia de } 70.5 \% \text {, } \\
\text { mientras que para dependencia de alcohol fue de } 37.8 \% \text {. }\end{array}$ \\
\hline & & $\begin{array}{l}\text { Tener un trastorno mental aumentó } 1.62 \text { ( } 95 \% \text { IC 0.83-3.16) la } \\
\text { probabilidad de presentar dependencia de drogas. }\end{array}$ \\
\hline
\end{tabular}


prevalencia de entre 50 y $70 \%$ de TCS (Baillargeon et al., 2009; Butler, Indig, Allnutt, \& Mamoon, 2011), mientras que para OTP puede llegar a ser de entre 11 y $42.7 \%$ (Baillargeon et al., 2009; Butler et al., 2011). Con respecto a la PD, se han encontrado prevalencias entre $6 \mathrm{y}$ 43.3\% (Baillargeon et al., 2010; Butler et al., 2011; Fazel \& Seewald, 2012; Lukasiewicz et al., 2009).

Entre los OTP más prevalentes en personas en prisión con TCS, se encuentran los trastornos de personalidad; destacan el trastorno antisocial de la personalidad, los trastornos psicóticos, de ansiedad, del estado de ánimo y por déficit de atención (Butler et al., 2011; Casares-López et al., 2010; Fazel \& Baillargeon, 2011; Fazel \& Danesh, 2002; Fazel \& Seewald, 2012; Fazel \& Yu, 2011; Lukasiewicz et al., 2009; Konstenius et al., 2015; Mundt et al., 2013). En la Tabla 2 se presentan algunos estudios observacionales específicos en patología dual en entornos penitenciarios.

Sobre los posibles factores de riesgo, la literatura reporta que condiciones comunes en el ambiente carcelario como poca higiene, hacinamiento, agresión entre reclusos y personal, aislamiento y falta de estimulación pueden tener un efecto debilitante en la salud mental, lo que contribuye al empeoramiento o la aparición de trastornos psiquiátricos (Shaw et al., 2009). Si se suma esto a hallazgos sobre la alta relación entre las conductas criminales y el consumo de sustancias (Black, Gunter, Loveless, Allen \& Sieleni, 2010; Pickard \& Fazel, 2013), se cuenta con argumentos para considerar que las personas en prisión son altamente vulnerables a presentar PD. También se ha encontrado que el número de sustancias consumidas es un factor predictor de PD (Villagrá, Fernández, García-Vega, \& González-Menéndez, 2013).

El género también juega un papel importante en el riesgo y prevalencia de la PD en esta población, siendo más común su ocurrencia en mujeres que en hombres (Baillargreon et al., 2010; Butler et al., 2011; Villagrá et al., 2013). Respecto a las diferencias en las prevalencias, resalta que mientras en reclusos hombres son más frecuentes los trastornos de ansiedad, psicóticos y antisocial de la personalidad, concurrentes con los trastornos por consumo de alcohol y por uso de cannabis, en las mujeres se presentan más los trastornos afectivos, de alimentación, por déficit de atención, de estrés postraumático y límite de la personalidad, concurrentes con el consumo de alcohol y estimulantes (Butler et al., 2011; Konstenius et al., 2015; Martínez-Lanz \& Fernández Díaz, 2013; Zlotnick et al., 2008). Sobre diferencias de género en factores de riesgo, se ha visto que en hombres el abuso físico en la infancia predice más la PD, mientras que en las mujeres, el abuso sexual tiene un mayor valor predictivo (Villagrá et al., 2013). También se ha documentado que entre las mujeres reclusas existe una mayor probabilidad de presentar conductas suicidas (Lukasiewicz et al., 2009) y se reporta una mayor violencia doméstica que en los hombres (Martínez Lanz et al., 2013).

Similar a lo observado en población indigente, la mayoría de los datos epidemiológicos sobre trastornos mentales en cárceles se obtiene de investigaciones realizadas en países de alto ingreso. En una revisión sistemática realizada por Fazel y Seewald (2012) se encontró que sólo 9\% de los estudios incluidos fueron realizados en países de mediano y bajo ingreso, donde la prevalencia de depresión (22.5\%) y psicosis (5.5\%) en reclusos fue mayor que en los países de alto ingreso ( $10 \%$ y $3.5 \%$, respectivamente). Adicionalmente, varios de los estudios llevados a cabo en estos países se han realizado con muestras pequeñas o provenientes de un número reducido de prisiones, lo que limita su representatividad (Cravioto, Medina-Mora, Rosa, Galván, \& Tapia-Conyer, 2003; Martínez-Lanz, Ocampo, Beyruti, \& de la Torre., 2012; Martínez-Lanz \& Fernández Díaz, 2013).

\section{Implicaciones de salud pública}

Diversos estudios han asociado los trastornos mentales con dificultades de ajuste al ambiente penitenciario, lo cual aumenta el riesgo de presentar abuso y padecer violaciones a los derechos humanos, asimismo dificulta la reintegración social e incrementa las probabilidades de indigencia o reingreso a la cárcel (Mundt et al., 2013; Colmenares-Bermúdez, Romero Mendoza, Rodríguez Ruiz, Durand-Smith, \& Saldívar Hernández, 2007; Fazel \& Seewald, 2012; McNiel, Binder, \& Robinson, 2005). Se ha documentado que es más probable que los reclusos con PD presenten múltiples encarcelamientos o les sea revocada la libertad condicional, comparados con quienes no tienen PD (Baillargeon et al., 2009; Baillargeon et al., 2010; Black et al., 2010; Pickard \& Fazel, 2013). Otras preocupaciones en el manejo del PD en cárceles es su asociación con conductas negativas exacerbadas (Houser \& Welsh, 2014) y con una mayor probabilidad de presentar episodios de violencia durante el internamiento (Wood \& Buttaroo, 2013).

Para asegurar el adecuado tratamiento de la salud mental de esta población, se recomienda contar con modelos colaborativos entre los sistemas correccionales y de salud -en coordinación con organizaciones no gubernamentales-, que contemplen la prevención y la atención conjunta de los TCS y OTP en todos los reclusos; es crucial la derivación en los primeros meses tras salir de prisión, para asegurar una mayor utilización de servicios de atención (Hartwell et al., 2013; Shackman \& Price, 2013). Aunque se ha reportado que los reclusos 
Tabla 2

Estudios observacionales sobre patología dual de personas en prisión

\begin{tabular}{|c|c|c|}
\hline Autores & Muestra y evaluación & Hallazgos \\
\hline $\begin{array}{l}\text { Baillargreon et al. } \\
(2010)\end{array}$ & $\begin{array}{l}61,248 \text { registros de reclusos que } \\
\text { comenzaron a cumplir sentencia } \\
\text { entre } 2000 \text { y } 2006 \text {. }\end{array}$ & $\begin{array}{l}\text { 7.4\% presentó un trastorno psiquiátrico del Eje I coocurrente con TCS; } \\
\text { 3.1\% un trastorno psiquiátrico solamente; y } 52.3 \% \text { sólo TCS. } \\
\text { La prevalencia de PD fue mayor en mujeres ( } 15.7 \% \text { ) que en hombres } \\
\text { (5.9); y en personas encarceladas por crímenes violentos ( } 8.6 \% \text { ) y con } \\
\text { sentencias de dos años o más }(6.0 \%) \text {. } \\
\text { La PD aumentó el riesgo de contar con múltiples encarcelamientos, } \\
\text { tanto en comparación con aquellos con sólo OTP (OR = 1.5, 95\% IC 1.1, } \\
\text { 2.2), como con quienes sólo presentan TCS (OR = 2.3, 95\% IC 1.9, 2.7). } \\
\text { Los OTP más prevalentes entre aquellos con TCS fueron la } \\
\text { esquizofrenia (62.8\%), el trastorno bipolar ( } 57.8 \% \text { ) y el trastorno } \\
\text { depresivo mayor (54.0\%). }\end{array}$ \\
\hline Butler et al. (2011) & $\begin{array}{l}\text { 1,478 individuos admitidos en el } \\
\text { sistema correccional, ya sea en } \\
\text { separos o en cárceles. } \\
\text { Diagnósticos CIE-10 en los } \\
\text { últimos } 12 \text { meses (Encuesta } \\
\text { Nacional Australiana de Salud } \\
\text { Mental y Bienestar). }\end{array}$ & $\begin{array}{l}\text { 29\% de la muestra presentó PD en los últimos } 12 \text { meses; siendo esta } \\
\text { más prevalente en mujeres ( } 46 \% \text { ) que en hombres (25\%). } \\
\text { Las prevalencias de OTP en las personas con TCS fueron las siguientes: } \\
\text { trastornos psicóticos ( } 76.6 \% \text { hombres, } 74.2 \% \text { mujeres), trastornos } \\
\text { afectivos ( } 65 \% \text { hombres, } 70.7 \% \text { mujeres) y trastornos de ansiedad ( } 66 \% \\
\text { hombres, } 75.2 \% \text { mujeres). } \\
\text { Los hombres con trastornos de ansiedad mostraron mayor probabilidad } \\
\text { de tener un trastorno por uso de alcohol y cannabis. Es más probable } \\
\text { que las mujeres con trastornos afectivos presenten trastorno por uso de } \\
\text { alcohol y estimulantes. }\end{array}$ \\
\hline $\begin{array}{l}\text { Casares-López et } \\
\text { al. (2010) }\end{array}$ & $\begin{array}{l}143 \text { personas recluidas en un } \\
\text { centro penitenciario. } \\
\text { Mini entrevista neuropsiquiátrica } \\
\text { internacional (MINI). }\end{array}$ & $\begin{array}{l}\text { 35\% de la muestra cumplió criterios para PD actual. Los OTP más } \\
\text { prevalentes en aquellos con PD fueron los trastornos ansiosos ( } 83.3 \%) \text {, } \\
\text { los afectivos ( } 79.2 \%) \text { y los psicóticos }(0.8 \%) \text {. } \\
\text { Entre los reclusos con PD se identificaron mayores prevalencias de } \\
\text { maltrato físico ( } 64.5 \%) \text { y psicológico ( } 64.5 \% \text { ) en la infancia; además de } \\
\text { que el maltrato físico aumenta siete veces el riesgo de presentar PD } \\
\text { (OR }=7,22, \text { IC } 95 \%, 2,33-22,33) \text {. }\end{array}$ \\
\hline $\begin{array}{l}\text { Lukasiewicz et al. } \\
\text { (2009) }\end{array}$ & $\begin{array}{l}998 \text { reclusos de } 23 \text { instituciones } \\
\text { penales distintas. } \\
\text { Mini entrevista neuropsiquiátrica } \\
\text { internacional (MINI) y } \\
\text { diagnóstico realizado por } \\
\text { psiquiatra. }\end{array}$ & $\begin{array}{l}26.3 \% \text { de la muestra presentó PD. } 90 \% \text { de los prisioneros con TCS } \\
\text { presentaron PD; mientras que } 38 \% \text { de la muestra con OTP presentó un } \\
\text { TCS concurrente. } \\
\text { La PD estuvo asociada con un mayor riesgo de suicidio (O\$ } 5.7 ; 95 \% \text { IC } \\
\text { 1.7-4.6). } \\
\text { Los trastornos de ansiedad fueron los más prevalentes ( } 79.7 \% \text { ), } \\
\text { seguidos por los trastornos del estado de ánimo ( } 73.8 \% \text { ) y los trastornos } \\
\text { psicóticos }(30.9 \%) \text {. }\end{array}$ \\
\hline Mundt et al. (2013) & $\begin{array}{l}1008 \text { reclusos de siete } \\
\text { instituciones penales distintas. }\end{array}$ & $\begin{array}{l}\text { Las concurrencias más prevalentes entre la muestra con uso de drogas } \\
\text { ilegales fueron los trastornos de ansiedad y explosivo intermitente } \\
\text { (16.7\%), los trastornos afectivos (12.1\%) y el déficit de atención (10.6\%). } \\
\text { En la muestra con trastorno depresivo mayor, las concurrencias más } \\
\text { prevalentes fueron los trastornos de ansiedad (43.5\%), los TCS (22.8\%), } \\
\text { el trastorno explosivo intermitente (18.9\%) y el déficit de atención } \\
(7.2 \%) \text {. }\end{array}$ \\
\hline
\end{tabular}


usan más los servicios de salud mental después de liberados (Hartwell et al., 2013), se enfrentan a múltiples barreras de tratamiento, particularmente las mujeres (Romero Mendoza, Saldívar, Loyola, Rodríguez, \& Galván, 2010; Romero Mendoza, 2010). Entre los principales obstáculos identificados se encuentran el estigma, la carga de enfermedad, las dificultades para mantener la abstinencia, la carencia de redes sociales de apoyo y las creencias sobre la sustancia o la condición de salud mental (Hartwell et al., 2013; Romero Mendoza et al., 2010); además de la falta de sostenibilidad de los servicios de salud mental en prisión (Shackman \& Price, 2013).

Por último, se ha reconocido que contar con herramientas adecuadas de detección y evaluación de trastornos mentales en el contexto penitenciario es una estrategia viable y pertinente para atender las necesidades de salud mental de esta población (Casares-López et al., 2011; Martin, Colman, Simpson, \& McKenzie, 2013), pues un tratamiento adecuado dentro de prisión podría facilitar la retención en servicios de atención posteriores.

\section{Personas con VIH, VHB y VHC}

\section{Epidemiología}

Desde hace más de dos décadas, la concurrencia de PD con VIH, VHB y VHC, también conocido como diagnóstico triple, se ha abordado como un problema que requiere atención especial (Batki, 1990; Ferrando \& Batki, 2000). En la literatura se ha reportado que entre las personas con PD o trastornos mentales severos, la prevalencia de VIH, VHB y VHC es más del doble en comparación con las personas que sólo presentan un trastorno, además de que muestran una prevalencia entre cinco y 12 veces mayor de trastornos mentales que la población general (Dausey \& Desai, 2003; McKinnon \& Rosner, 2000; Ro- senberg et al., 2001; Rosenberg, Drake, Brunette, Wolford, \& Marsh, 2005). Estudios realizados en poblaciones de personas con VIH, VHB y VHC encontraron una prevalencia de PD o trastornos mentales de entre 10 y 28\%; así como prevalencias de hasta $86 \%$ de TCS (Burman et al., 2001; Dausey \& Desay, 2003; Galván, Burnam, \& Bing, 2003; Gupta, Kumar, \& Garg, 2013; Whetten et al., 2005; Yovtcheva, Rifai, Moles, \& Van Der Linden, 2001). Finalmente, el último estudio global sobre carga de enfermedad reporta que las personas que utilizan drogas inyectables presentan altas prevalencias de PD (Mackesy-Amiti, Donenberg, \& Ouellet, 2012); se sabe, además, que la PD es uno de los factores de riesgo más importantes para contraer VIH y VHC (Degenhardt \& Hall, 2012).

Por otra parte es conocido que entre los trastornos mentales mayormente asociados al riesgo de infección, además de los TCS, se encuentran los trastornos psicóticos, los del estado de ánimo, el déficit de atención y los de ansiedad (Rosenberg et al., 2001; Yovtcheva et al., 2001; Dunne, Hearn, Rose, \& Latimer, 2014). En la Tabla 3 se presentan algunos hallazgos de estudios observacionales sobre la concurrencia de TCS y OTP en esta población.

Adicionalmente, se han propuesto diversos modelos explicativos para esta condición clínica, que toman en consideración efectos directos (por ejemplo labilidad afectiva, impulsividad o déficit atencional) e indirectos (por ejemplo vivir en la calle) de los OTP en las personas con TCS, los cuales pueden incrementar las conductas de alto riesgo y resultar en tasas elevadas de infección (Dunne et al., 2014; McKinnon et al., 1996; Fazel et al., 2014). Entre las conductas de riesgo asociadas se puede incluir el uso de drogas inyectables y compartir jeringas, así como tener relaciones sexuales de alto riesgo (por ejemplo sexo sin protección, tener sexo bajo

Tabla 3

Estudios observacionales sobre patología dual en población indigente

\begin{tabular}{|c|c|c|}
\hline Autores & Muestra y evaluación & Hallazgos \\
\hline \multirow[t]{2}{*}{$\begin{array}{l}\text { Dausey \& Desai } \\
\text { (2003) }\end{array}$} & $\begin{array}{l}200 \text { pacientes VIH positivos } \\
\text { que reciben tratamiento para } \\
\text { adicciones. }\end{array}$ & $\begin{array}{l}43.0 \% \text { de la muestra presentó cualquier trastorno psiquiátrico } \\
\text { concurrente. }\end{array}$ \\
\hline & $\begin{array}{l}\text { Autorreporte de historia de } \\
\text { trastornos mentales. }\end{array}$ & $\begin{array}{l}\text { Los pacientes VIH positivos tuvieron casi } 2.5 \text { veces mayores } \\
\text { probabilidades de presentar PD que los pacientes VIH negativos. }\end{array}$ \\
\hline \multirow[t]{2}{*}{$\begin{array}{l}\text { Gupta et al. } \\
\text { (2013) }\end{array}$} & $\begin{array}{l}100 \text { pacientes VIH positivos que } \\
\text { recibían terapia antirretroviral. }\end{array}$ & $\begin{array}{l}26.0 \% \text { de la muestra total presentó PD ( } 56 \% \text { de quienes presentaban } \\
\text { TCS). }\end{array}$ \\
\hline & $\begin{array}{l}\text { Cédula neuropsiquiátrica de } \\
\text { evaluación clínica. }\end{array}$ & $\begin{array}{l}\text { El trastorno depresivo mayor fue el más común ( } 28 \% \text { de quienes } \\
\text { presentaban TCS), seguido por el trastorno de ansiedad generalizada } \\
(16 \%) \text {. }\end{array}$ \\
\hline
\end{tabular}


el efecto de sustancias e intercambiar drogas por sexo) (Carey, Carey, \& Kalichman, 1997; Dausey \& Desai, 2003; Dunne et al., 2014; McKinnon, 1996; King, Kidorf, Stoller, \& Brooner, 2000).

Con respecto al género, se han identificado mayores prevalencias de PD en mujeres con respecto a los hombres (Dausey \& Desai, 2003), así como de coinfección (VIH+VHC) y PD (Rosenberg et al., 2005). Por otro lado, condiciones como el estatus migratorio o el trabajo sexual aumentan significativamente el riesgo de infección, coinfección y la ocurrencia de TCS y OTP (Platt et al., 2012). Un estudio realizado en mujeres con VIH en recuperación encontró que la concurrencia de trastornos por uso de opiáceos y cocaína en esta población está asociado con un empeoramiento en la salud mental y con sintomatología física del VIH (McCabe, Feaster, \& Mitrani, 2014).

Implicaciones de salud pública

La conexión entre los trastornos mentales y el VIH, VHB o VHC tiene importantes implicaciones médicas y psicológicas que es necesario considerar para su prevención y tratamiento conjuntos. Por un lado, los TCS comprometen la respuesta al tratamiento de los demás trastornos mentales y las enfermedades infecciosas (Dausey \& Desai, 2003; King et al., 2000; Mayston, Kinyanda, Chishinga, Prince, \& Patel, 2012); pero por el otro, se ha documentado que contar con una enfermedad infecciosa también tiene consecuencias en la salud mental de quienes la padecen (por ejemplo exacerbación de síntomas depresivos, distrés psicológico y problemas de sueño) (Collins, Holman, Freeman \& Patel, 2006; McCabe et al., 2014).

El uso de sustancias por personas con VIH provoca consecuencias adversas que pueden ser muy graves, como un decremento en el autocuidado, neumonía y abscesos en la piel; además, el efecto directo de las sustancias psicoactivas puede suprimir varios aspectos de las funciones inmunológicas (Ferrando \& Batki, 2000; McCabe et al., 2014). Adicionalmente, los trastornos mentales en esta población pueden interferir en el curso y el tratamiento de dichas enfermedades (Yovtcheva et al., 2001), aumentar la probabilidad de que las personas presenten conductas de riesgo de infección a terceros y retrasar el tiempo de acceso a tratamiento por desconocimiento del estado de infección (Campollo et al., 2012; White et al., 2007).

Debido a lo anterior, se requiere un enfoque de tratamiento integrado que englobe la atención psicosocial y el manejo farmacológico (Ferrando \& Batki, 2000); para ello es necesario hacer más accesibles los servicios de salud mental en escenarios comunitarios (por ejemplo atención primaria y servicios de trabajo social) y redu- cir, de esta forma, las barreras de atención a las que se enfrentan los usuarios de sustancias infectados, como el estigma y los riesgos asociados a la doble carga de enfermedad (Burnam et al., 2001; Collins et al.,2006).

Es probable que el diagnóstico y el tratamiento psiquiátrico de esta población se compliquen por los daños neuropsiquiátricos (que pueden ser a largo plazo, por el consumo de sustancias de abuso, o secundarios a un traumatismo psíquico) (Batki et al., 1996; Stern, 1994); además, enfermedades como el VIH tienden a asociarse con anormalidades cognitivas, motoras y de comportamiento, tanto en las etapas iniciales como en las avanzadas (Ferrando \& Batki, 2000; Collins et al., 2006). Finalmente, se ha documentado que los trastornos mentales dificultan la adherencia al tratamiento retroviral, especialmente en países de mediano y bajo ingreso (Collins et al., 2006; Mayston et al., 2012).

Entre las intervenciones psicosociales para el tratamiento de la salud mental de esta población se incluyen los grupos de ayuda mutua, dado su potencial para reducir el aislamiento social (Ferrando \& Batki, 2000). Asimismo, se han reportado resultados prometedores para intervenciones como la psicoeducación y las cognitivo-conductuales grupales, orientadas a asistir en estrategias de afrontamiento y manejo del VIH; también ha resultado eficiente la identificación o exploración de la salud mental por parte de no-profesionales en la comunidad (Collins et al., 2006). En la actualidad, se debate la incorporación de tratamientos sustitutivos y aproximaciones basadas en la reducción del daño, particularmente en usuarios de drogas intravenosas, entre quienes la prevalencia de patología dual y el riesgo de infección es mayor (por ejemplo intercambio de jeringas, tratamiento de mantenimiento por metadona, provisión de espacios seguros para el consumo y programas de alojamiento) (Hawk \& Davis, 2012; Aspinall et al., 2014).

\section{Mujeres}

\section{Epidemiología}

La literatura indica que las mujeres son particularmente vulnerables a las consecuencias del consumo de alcohol y drogas, lo cual se relaciona con factores fisiológicos, psicológicos y socioculturales (Medina-Mora, 2011).

Estudios epidemiológicos internacionales (Kessler et al., 1997) reportan que las mujeres con abuso o dependencia del alcohol presentan tasas más altas de OTP, comparadas con los varones con la misma problemática (65\% vs. 44\%) (Zilberman, Tavares, Blume, \& Guebaly, 2003). Entre los trastornos psiquiátricos identificados como más prevalentes en las mujeres con PD, se han identificado los trastornos afectivos (excepto el trastor- 
no bipolar tipo I) y los trastornos de ansiedad; así como mayor adicción a hipnosedantes, con respecto a los hombres (Miquel, Roncero, López-Ortiz \& Casas, 2010; Miquel et al., 2013). Por otro lado, aquellas mujeres con trastornos psicóticos y consumo concurrente de sustancias tienen mayores dificultades en el pronóstico de tra- tamiento, pues presentan, con respecto a los hombres, un mayor número de hospitalizaciones, mayor severidad en los síntomas positivos, mayor exposición a algún tipo de abuso y disminución en el funcionamiento global (Miquel et al., 2013). La Tabla 4 presenta algunos hallazgos relevantes sobre PD en mujeres.

Tabla 4

Estudios observacionales sobre patología dual en mujeres

\begin{tabular}{cll}
\hline \multicolumn{1}{c}{ Autores } & \multicolumn{1}{c}{ Muestra y evaluación } & \multicolumn{1}{c}{ Hallazgos } \\
\hline Chen et al. (2011) & 465 personas internadas en & Las mujeres tienen mayor probabilidad de cumplir con criterios \\
& centros de tratamiento para las & diagnósticos para un trastorno psiquiátrico (73.7\% mujeres contra \\
& adicciones. & $55.4 \%$ hombres).
\end{tabular}

Entrevista clínica estructurada para el DSM-IV (SCID-I).

Drapalski et al.(2011)

Steingrímsson et al. (2012)

Miquel et al. (2013)

Morse et al. (2015)
312 personas provenientes de dos muestras con trastornos mentales severos y trastorno por uso de cocaína.

Entrevista clínica estructurada para el DSM-IV (SCID-I) y Escala de Síntomas Positivos y Negativos para Psicosis (PANSS).

\section{5,192 registros de} hospitalizaciones psiquiátricas entre 1983 y 2007.

Diagnósticos CIE-9 y CIE-10 realizados por un psiquiatra durante la admisión a tratamiento. 85 pacientes diagnosticados con Patología Dual (PD).

Entrevista clínica estructurada para el DSM-IV (SCID-I).

1,317 pacientes con PD en tres facilidades de tratamiento residencial.

Índice de Severidad de la Adicción (ASI).
Las mujeres mostraron mayor prevalencia de dependencia de cocaína, concurrente con cualquier trastorno psiquiátrico (50.8\%).

Entre los OPT más prevalentes en mujeres con TCS están los trastornos del estado de ánimo (32.6\%) y ansiedad (31.1\%), los síntomas psicóticos (13.1\%) y el trastorno límite de la personalidad (27.7\%).

Fue mayor la prevalencia de mujeres con dependencia a dos sustancias o más (32.6\%).

La prevalencia de trastornos afectivos concurrentes es mayor tanto en mujeres que buscan tratamiento (58\%) como en aquellas que no solicitan atención (51\%), con respecto a los hombres.

El porcentaje de mujeres con trastorno psicótico concurrente fue mayor en la muestra que no busca tratamiento (47\%) que en aquella que busca atención (36\%).

Las mujeres mostraron mayor probabilidad con reporte de abuso sexual a lo largo de la vida que los hombres.

La edad promedio de admisión decreció en mayor medida en las mujeres (48.9 a 41.7) que en los hombres (42.5 a 39.8).

La proporción de trastornos por consumo de alcohol cambió de 4.2 hombres por mujer en 1983, a 1.5 hombres por mujer en 2007.

Las mujeres reportaron con mayor frecuencia los hipnosedantes como droga de preferencia (60.2\%), con respecto a los hombres.

Aunque las mujeres reportan inicio de consumo a una edad más avanzada (media: 22.1) con respecto a los hombres (18 años), no hubo diferencias significativas por género en longitud de TCS.

Las mujeres presentaron, en mayor porcentaje que los hombres, prevalencias de trastorno depresivo mayor (82.7\%), trastorno de ansiedad (87.8\%), trastorno por déficit de atención (1.9\%) y trastornos de la alimentación (1.3\%).

Los hombres con PD presentaron mayor dificultad para mantenerse en tratamiento que las mujeres. 
En lo correspondiente a la etiología de la PD, se ha encontrado que entre 55 y $99 \%$ de las mujeres con PD tiene historias de trauma y abuso a lo largo de la vida (Daigre et al., 2015; Miller \& Downs, 1995; Najavits, Weiss, \& Shaw, 1997) y presenta mayor probabilidad de haber sido víctima de violencia, que las mujeres con sólo una condición psicopatológica (Alexander, 1996); asimismo, presentan mayores prevalencias de abuso sexual en la infancia (13.5\%), con respecto a los hombres (2.5\%) (Molnar, Buka, \& Kessler, 2001).

Si se considera que la literatura reporta que las experiencias traumáticas en la infancia son un factor de riesgo importante para la aparición de síntomas psicopatológicos en la adultez en ambos sexos (Felitti et al., 1998; Foege, 1998; Weiss \& Wagner, 1998) -además de la relación bidireccional entre la violencia y la PD $(\mathrm{McHu}-$ go et al., 2005)-, se puede decir que las mujeres muestran marcadamente mayor vulnerabilidad a presentar esta condición.

Implicaciones de salud pública

Las mujeres se enfrentan a mayores barreras de tratamiento para los trastornos mentales que los hombres; entre las que se han identificado se cuentan la detección tardía del problema, el ensombrecimiento diagnóstico, la carencia de centros de tratamiento sensibles a las necesidades de las mujeres y el estigma social. Asimismo, la vulnerabilidad acumulada contribuye al desarrollo de otros problemas asociados como riesgo de perder la custodia de los hijos, rechazo de la pareja y pérdida del apoyo familiar (Galván \& Marín Navarrete, 2010; Grella, 1996; Romero Mendoza, 2010).

Debido a que la violencia, el trauma, la opresión y el estigma son temas comunes en la realidad de las mujeres con esta condición, diversos autores indican que el desarrollo e implementación de tratamientos sensibles a diferencias de género podrían empoderarlas para facilitar que asuman un papel más activo en el tratamiento (Egeland \& Erickson, 1990; Miquel et al., 2013). Las observaciones han demostrado que ellas presentan mejores respuestas a las intervenciones diseñadas específicamente para mujeres con TCS y OTP (Morse, MacMaster, Choy, \& Adams; 2015; Ruglass, Hien, Hu \& Campbell, 2014). Por otro lado, a pesar de que las mujeres con PD suelen entrar a tratamiento una vez que presentan mayor severidad en su sintomatología y disfunción en diversas áreas, presentan mayor retención en tratamiento que los hombres con PD (Morse et al., 2015).

Por último, las mujeres embarazadas requieren consideración especial (Grella, 1996), ya que la prevalencia y la vulnerabilidad ante la PD es mayor que en no embarazadas; diversas revisiones sistemáticas sobre salud y cuidados perinatales han identificado que esta población muestra una particular vulnerabilidad a presentar trastornos de ansiedad, depresión y trastornos somáticos; además de que el consumo de sustancias y los trastornos mentales incrementan significativamente el riesgo de sufrir complicaciones durante el parto o de que se presenten condiciones que comprometan la salud del producto (por ejemplo síndrome alcohólico fetal) (Bennet, Einarson, Taddio, Koren, \& Einarson, 2004; Fisher et al., 2012; Ross, McLean, \& Psych, 2006; Shaw, Levitt, Wong, \& Kaczorowski, 2006).

\section{Adultos mayores}

\section{Epidemiología}

El consumo de sustancias en los adultos mayores es una realidad subdiagnosticada (Roncero, Bachiller, Ros, \& Casas, 2011; Mendoza-Meléndez et al., 2015). Una revisión realizada por Bartels, Blow, Van Citters y Brockmann (2006) sobre PD en población de adultos mayores reporta que una historia de abuso de sustancias a lo largo de la vida incrementa el riesgo de presentar un trastorno mental en la vejez, mientras que de manera inversa, la presencia de trastornos mentales también está asociada con un mayor riesgo de presentar un TCS. La misma revisión señala prevalencias considerables de TCS (4\%-11\%) en clínicas de psicogeriatría, las cuales aumentan hasta $37.6 \%$ en contextos de hospitalización, donde es más frecuente el abuso concurrente de alcohol (Bartels et al., 2006). De igual manera, estudios realizados en pacientes geriátricos tratados por TCS indican una prevalencia de OTP de entre 21 y $66 \%$, siendo la concurrencia entre trastornos por uso de alcohol y depresión, la más frecuente en esta población (Blow, Cook, Booth, Falcon \& Friedman, 1992; Caputo et al., 2012; Brennan, Schute, \& Moos, 2005). También se ha identificado que la presencia conjunta de trastornos por uso de alcohol, trastornos ansiosos y trastorno depresivo tiene una relación causal con $70 \%$ de los suicidios reportados en esta población, así como con episodios de violencia doméstica, dificultades en el funcionamiento social y mayor nivel de discapacidad (Caputo et al., 2012; Gum, King-Kallimanis, \& Kohn, 2009; Salmon \& Forrester, 2012). Algunos estudios relevantes sobre concurrencia de TCS y OTP en adultos mayores se presentan en la Tabla 5.

Con respecto a la etiología de la PD en esta población, se ha reportado que factores como la muerte de seres queridos, las enfermedades médicas, la pérdida del trabajo y el retiro, pueden influir negativamente en el estado de ánimo y en el consumo de alcohol (Blow \& Barry, 2003; Blow, Serras, \& Barry, 2007). Un estudio 
Tabla 5

Estudios observacionales sobre patología dual en personas mayores

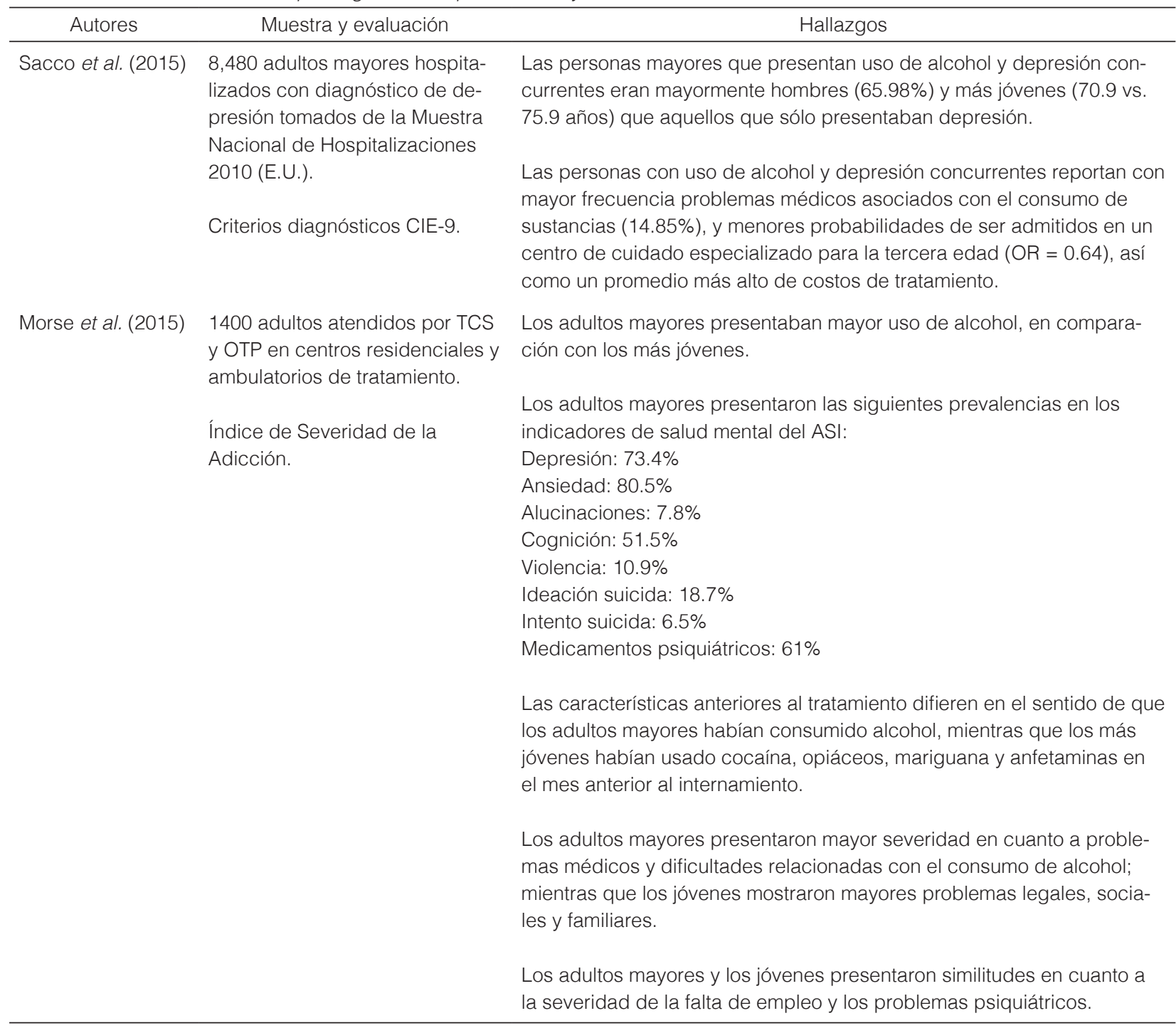

realizado en población general de la tercera edad en México identificó que el diagnóstico de una o más enfermedades concurrentes aumenta las probabilidades de presentar consumo de sustancias (Mendoza-Meléndez et al., 2015).

Implicaciones de salud pública

Los TCS y OTP en esta población incrementan el riesgo de padecer enfermedades crónico-degenerativas (como diabetes mellitus, cataratas, ceguera, glaucoma, úlcera duodenal y cáncer, entre otras), enfermedades infecciosas, problemas de sueño y de memoria, y demencias (Aguilar-Navarro \& Ávila-Funes, 2007; Blow \&
Barry, 2003; Blow et al., 2007; Caputo et al., 2012; Mendoza-Meléndez et al., 2015).

Por otro lado, se ha encontrado que los adultos mayores muestran alta frecuencia de polifarmacia, así como un riesgo mayor de uso inapropiado de medicamentos (Blow \& Barry, 2003), lo que aunado al consumo de alcohol y a una disminución de la función renal, aumenta el riesgo de presentar eventos adversos (por ejemplo interacción entre fármacos), detrimento de la calidad de vida y deterioro cognitivo (Antonelli-Incalizi, Corsonello, Pedone, Corica, \& Carbonin, 2005; Mendoza-Meléndez et al., 2015; Sim, Hulse, \& Khong, 2004). Adicionalmente, la incidencia de complicaciones médicas y neurológicas durante el síndrome de abstinencia 
por alcohol es más elevada en los adultos mayores que en los más jóvenes; asimismo, estas personas son más vulnerables a presentar enfermedad hepática alcohólica (Caputo et al., 2012).

Además, un estudio realizado con usuarios de los servicios de salud para veteranos encontró que los pacientes de edad avanzada (mayor a 55 años) con PD usan menos los servicios de tratamiento y requieren estancias más extendidas en hospitalización (Kerfoot, Petrakis, \& Rosenheck, 2011). En cuanto al tratamiento, las intervenciones terapéuticas breves (Blow \& Barry, 2003; Blow et al., 2007), la terapia cognitivo conductual, la terapia comunitaria (D'Agostino, Barry, Blow, \& Podgorski, 2006) y la participación en grupos de ayuda mutua, en conjunto con el tratamiento farmacológico (Oslin, Liberto, O'Brien, Krois, \& Norbeck, 1997; Satre, Blow, Chi, \& Weisner, 2007), han mostrado resultados promisorios en esta población. Finalmente se sabe que en algunos casos, la prevención de recaídas puede resultar mejor en adultos mayores que en pacientes más jóvenes, pues los primeros se mantienen en abstinencia hasta después de cuatro años de haber recibido el tratamiento (Satre et al., 2004; Satre et al., 2007).

\section{DISCUSIÓN Y CONCLUSIONES}

La presente revisión integra hallazgos relevantes sobre la PD en poblaciones especiales de personas en situación de indigencia, en prisión, con VIH, VHB y VHC, mujeres y adultos mayores. La evidencia encontrada sobre estas poblaciones indica que, además de presentar diferencias importantes en prevalencias de PD respecto a la población general, su ocurrencia está asociada con mayores tasas de mortalidad por enfermedades crónicas, transmisibles y violencia. Asimismo, se encontró que la pertenencia a una de estas poblaciones está asociada a la probabilidad de pertenecer a otra (por ejemplo las personas liberadas de prisión con PD presentan alto riesgo de indigencia).

Lo anterior debe tomarse en consideración al formular políticas públicas. Los planes diseñados de manera específica para atender las necesidades particulares de estas poblaciones en forma integral demuestran resultados más promisorios, no sólo en la atención de la salud mental, sino también en la disminución de sus niveles de marginación y en la mejora de su salud general. Asimismo, debe considerarse que los sistemas de atención social dirigidos a estas poblaciones suponen una oportunidad para identificar de manera oportuna trastornos mentales que pasarían desapercibidos, así como para dar un seguimiento adecuado a su tratamiento (por ejemplo sistemas penitenciarios).
Es notable la necesidad de realizar investigaciones dirigidas específicamente al estudio de la PD en países de mediano y bajo ingreso. La evidencia incluida en esta revisión proviene en su mayoría de países de alto ingreso, donde las diferencias de contexto, los registros y el sistema de apoyo de las poblaciones especiales son diferentes a los implementados en poblaciones menos desarrolladas. También se requiere investigación con muestras más representativas, mayor precisión diagnóstica y el empleo de metodologías más sofisticadas, con el objetivo de contar con mayor calidad en los hallazgos. No obstante, hay que considerar que la marginación y la dificultad de acceso a estas poblaciones pueden representar un verdadero reto en la implementación de las investigaciones.

\section{Limitaciones}

La cantidad de literatura dirigida específicamente a la PD en estas cuatro poblaciones es limitada y se realiza con muestras reducidas, por lo que la información presentada debe tomarse con cautela. Otra limitación es la no inclusión de los trastornos por consumo de tabaco y el juego patológico, ambos incorporados como trastornos adictivos en la última edición del DSM-5. Finalmente, es necesario contar con más evidencia sobre PD en otras poblaciones especiales como adolescentes en riesgo, trabajadoras sexuales, indígenas, migrantes en tránsito, refugiados y otros grupos minoritarios.

\section{CONFLICTOS DE INTERÉS}

Los autores declaran no tener conflictos de interés sobre la información presentada.

\section{FUENTES DE FINANCIAMIENTO}

Ninguno.

\section{REFERENCIAS}

Aguilar-Navarro, S., \& Ávila-Funes, J. A. (2007). La depresión: particularidades clínicas y consecuencias en el adulto mayor. Gaceta Médica Méxicana, 143(2), 141-146.

Alexander, M. J. (1996). Women with co-occurring addictive and mental disorders: An emerging profile of vulnerability. American Journal of Orthopsychiatry, 66, 61-70.

Antonelli-Incalzi, R. A., Corsonello, A., Pedone, C., Corica, F., \& Carbonin, P. (2005). Depression and drug utilization in an elderly population. Therapeutics and Clinical Risk Management, 1(1), 55

Asante, K. O., Meyer-Weitz, A., \& Petersen, I. (2014). Substance use and risky sexual behaviours among street connected children and youth in Accra, Ghana. Moment, 5(8), 11. doi:10.1186/1747-597X-9-45 
Aspinall, E. J., Nambiar, D., Goldberg, D. J., Hickman, M., Weir, A., Van Velzen, E., ... Hutchinson, S. J. (2014). Are needle and syringe programmes associated with a reduction in HIV transmission among people who inject drugs: a systematic review and meta-analysis. International Journal of Epidemiology, 43(1), 235-248. doi:10.1093/ije/dyt243

Aubry, T., Tsemberis, S., Adair, C. E., Veldhuizen, S., Streiner, D., Latimer, E., ... Goering, P. (2015). One-year outcomes of a randomized controlled trial of housing first with ACT in five Canadian cities. Psychiatric Services, 66(5), 463-469.

Baillargeon, J., Penn, J. V., Knight, K., Harzke, A. J., Baillargeon, G., \& Becker, E. A. (2010). Risk of reincarceration among prisoners with co-occurring severe mental illness and substance use disorders. Administration and Policy in Mental Health and Mental Health Services Research, 37(4), 367-374. doi: 10.1007/ s10488-009-0252-9

Baillargeon, J., Williams, B.A., Mellow, J., Harzke, A.J., Hoge, S.K., Baillargeon, G., \& Greifinger, R.B. (2009). Parole revocation among prison inmates with psychiatric and substance use disorders. Psychiatric Services, 60(11): 1516-1521. doi:10.1176/ appi.ps.60.11.1516

Bartels, S. J., Blow, F. C., Van Citters, A. D., \& Brockmann, L. M. (2006). Dual diagnosis among older adults: Co-occurring substance abuse and psychiatric illness. Journal of Dual Diagnosis, 2(3), 9-30. doi:10.1300/J374v02n03_03

Batki, S. L. (1990). Drug abuse, psychiatric disorders, and AIDS. Dual and triple diagnosis. Western Journal of Medicine, 152(5), 547.

Batki, S. L., Ferrando, S. J., Manfredi, L., London, J., Pattillo, J., \& Delucchi, K. (1996). Psychiatric disorders, drug use, and medical status in injection drug users with HIV disease. The American Journal on Addictions, 5(3), 249-258.

Baxter, A. J., Patton, G., Scott, K. M., Degenhardt, L., \& Whiteford, H. A. (2013). Global epidemiology of mental disorders: what are we missing. PLoS One, 8(6), e65514. doi:10.1371/journal. pone.0065514

Beijer, U., Andreasson, S., Ågren, G., \& Fugelstad, A. (2011). Mortality and causes of death among homeless women and men in Stockholm. Scandinavian journal of public health, 39(2), 121127. doi:10.1177/1403494810393554

Bennett, H. A., Einarson, A., Taddio, A., Koren, G., \& Einarson, T. R. (2004). Prevalence of depression during pregnancy: systematic review. Obstetrics \& Gynecology, 103(4), 698-709. doi: 10.1097/01.AOG.0000116689.75396.5f

Black, D.W., Gunter, T., Loveless, P., Allen, J., \& Sieleni, B. (2010). Antisocial personality disorder in incarcerated offenders: Psychiatric comorbidity and quality of life. Annals of Clinical Psychiatry, 22(2): 113-120

Blow, F. C., \& Barry, K. L. (2003). Use and misuse of alcohol among older women. Alcohol Research and Health, 26(4), 308-315.

Blow, F.C., Cook, C.A., Booth, B.M., Falcon, S.P., \& Friedman, M. J. (1992). Age-related psychiatric comorbidities and level of functioning in alcoholic veterans seeking outpatiens treatmen. Hospital and Community Psychiatry, 43(10), 990-995.
Blow, F. C., Serras, A. M., \& Barry, K. L. (2007). Late-life depression and alcoholism. Current Psychiatry Reports, 9(1), 14-19. doi: 10.1007/s11920-007-0004-z

Borges, G., Medina-Mora, M. E., \& López-Moreno, S. (2004). El papel de la epidemiología en la investigación de los trastornos mentales. Salud Pública de México, 46(5), 451-463.

Brennan, P. L., Schutte, K. K., \& Moos, R. H. (2005). Pain and use of alcohol to manage pain: prevalence and 3 year outcomes among older problem and non problem drinkers. Addiction, 100(6), 777-786. doi:10.1111/j.1360-0443.2005.01074.x

Burnam, M. A., Bing, E. G., Morton, S. C., Sherbourne, C., Fleishman, J. A., London, A. S., ... Shapiro, M. F. (2001). Use of mental health and substance abuse treatment services among adults with HIV in the United States. Archives of General Psychiatry, 58(8), 729-736. doi:10.1001/archpsyc.58.8.729.

Butler, T., Indig, D., Allnutt, S., \& Mamoon, H. (2011). Co-occurring mental illness and substance use disorder among Australian prisoners. Drug and Alcohol Review, 30(2), 188-194. doi: 10.1111/j.1465-3362.2010.00216.x

Campollo, O., Roman, S., Panduro, A., Hernández, G., Díaz-Barriga, L., Balanzario, M. C., \& Cunningham, J. K. (2012). Non-injection drug use and hepatitis $C$ among drug treatment clients in west central Mexico. Drug and Alcohol Dependence, 123(1), 269272. doi:10.1016/j.drugalcdep.2011.11.009

Canavan, R., Barry, M. M., Matanov, A., Barros, H., Gabor, E., Greacen, T., ... Priebe, S. (2012). Service provision and barriers to care for homeless people with mental health problems across 14 European capital cities. BMC Health Services Research, 12(1), 222. doi:10.1186/1472-6963-12-222

Caputo, F., Vignoli, T., Leggio, L., Addolorato, G., Zoli, G., \& Bernardi, M. (2012). Alcohol use disorders in the elderly: a brief overview from epidemiology to treatment options. Experimental Gerontology, 47(6), 411-416. doi:10.1016/j.exger.2012.03.019

Carey, M. P., Carey, K. B., \& Kalichman, S. C. (1997). Risk for human immunodeficiency virus (HIV) infection among persons with severe mental illnesses. Clinical Psychology Review, 17(3), 271291.

Casares-López, M. J. C., González-Menéndez, A., Bobes-Bascarán, M. T., Secades-Villa, R., Martínez-Cordero, A., \& Bobes, J. (2011). Necesidad de evaluación de la patología dual en contexto penitenciario. Adicciones, 23(1), 37-44.

Casares-López, M. J. C., González-Menéndez, A., Torres-Lobo, M., Secades-Villa, R., Fernández-Hermida, J. R., \& Álvarez, M. (2010). Comparación del perfil psicopatológico y adictivo de dos muestras de adictos en tratamiento: en prisión y en comunidad terapéutica. International Journal of Clinical and Health Psychology, 10(2), 225-243.

Chen, K. W., Banducci, A. N., Guller, L., Macatee, R. J., Lavelle, A., Daughters, S. B., \& Lejuez, C. W. (2011). An examination of psychiatric comorbidities as a function of gender and substance type within an inpatient substance use treatment program. Drug and Alcohol Dependence, 118(2), 92-99. doi:10.1016/j.drugalcdep.2011.03.003 
Colmenares Bermúdez, E., Romero Mendoza, M. P., Rodríguez Ruiz, E. M., Durand-Smith, A. L., \& Saldívar Hernández, G. J. (2007). Female depression and substance dependence in the Mexico City penitentiary system [Depresión en mujeres y la dependencia a sustancias en el sistema penitenciario de la Ciudad de México]. Salud Mental, 30(6), 53-61.

Collins, P. Y., Holman, A. R., Freeman, M. C., \& Patel, V. (2006). What is the relevance of mental health to HIVIAIDS care and treatment programs in developing countries? A systematic review. AIDS, 20(12), 1571-1582. doi: http://doi.org/10.1097/01. aids.0000238402.70379.d4

Cravioto, P., Medina-Mora, M. E., Rosa, B. D. L., Galván, F., \& Tapia-Conyer, R. (2003). Patrones de consumo de heroína en una cárcel de la frontera norte de México: barreras de acceso a tratamiento. Salud Pública de México, 45(3), 181-190.

D’Agostino, C. S., Barry, K. L., Blow, F. C., \& Podgorski, C. (2006). Community interventions for older adults with comorbid substance abuse: the Geriatric Addictions Program (GAP). Journal of Dual Diagnosis, 2(3), 31-45. doi:10.1300/ J374v02n03_04

Daigre, C., Rodríguez-Cintas, L., Tarifa, N., Rodríguez-Martos, L., Grau-López, L., Berenguer, M., ... Roncero, C. (2015). History of sexual, emotional or physical abuse and psychiatric comorbidity in substance-dependent patients. Psychiatry Research, 229(3), 743-749. doi:10.1016/j.psychres.2015.08.008

Dausey, D. J., \& Desai, R. A. (2003). Psychiatric comorbidity and the prevalence of HIV infection in a sample of patients in treatment for substance abuse. The Journal of Nervous and Mental Disease, 191(1), 10-17.

Degenhardt, L., \& Hall, W. (2012). Extent of illicit drug use and dependence and their contribution to the global burden of disease. The Lancet, 379(9810), 55-70. doi:10.1016/S01406736(11)61138-0

Demyttenaere, K., Bruffaerts, R., Posada-Villa, J., Gasquet, I., Kovess, V., Lepine, J., ... Chatterji, S. (2004). Prevalence, severity and unmet need for treatment of mental disorders in the World Health Organization World Mental Health Surveys. The Journal of the American Medical Association, 291(21), 2581-2590. doi: 10.1001/jama.291.21.2581

Drapalski, A., Bennett, M., \& Bellack, A. (2011). Gender differences in substance use, consequences, motivation to change, and treatment seeking in people with serious mental illness. Substance Use \& Misuse, 46(6), 808-818. doi: 10.3109/10826084.2010.538460

Dunne, E. M., Hearn, L. E., Rose, J. J., \& Latimer, W. W. (2014). ADHD as a risk factor for early onset and heightened adult problem severity of illicit substance use: An accelerated gateway model. Addictive Behaviors, 39(12), 1755-1758. doi: 10.1016/j. addbeh.2014.07.009

Egeland, B., \& Erickson, M. F. (1990). Rising above the past: Strategies for helping new mothers break the cycle of abuse and neglect. Zero to Three, 11, 29- 35.
Fazel S., \& Baillargreon J. (2011). The health of prisoners. The Lancet, 377(9769), 956-965. doi:10.1016/S0140-6736(10)61053-7

Fazel, S. \& Danesh, J. (2002). Serious mental disorder in 23000 prisoners: a systematic review of 62 surveys. The Lancet, 359(9306), 545-550. doi:10.1016/S0140-6736(02)07740-1

Fazel, S., Geddes, J. R., \& Kushel, M. (2014). The health of homeless people in high-income countries: descriptive epidemiology, health consequences, and clinical and policy recommendations. The Lancet, 384(9953), 1529-1540. doi:10.1016/S01406736(14)61132-6

Fazel, S., Khosla, V., Doll, H., \& Geddes, J. (2008). The prevalence of mental disorders among the homeless in western countries: systematic review and meta-regression analysis. PLOS Med, 5(12), e225. doi: 10.1371/journal.pmed.0050225

Fazel, S., \& Seewald, K. (2012). Severe mental illness in 33588 prisoners worldwide: systematic review and meta-regression analysis. The British Journal of Psychiatry, 200(5), 364-373. doi: 10.1192/bjp.bp.111.096370

Fazel, S., \& Yu, R. (2011). Psychotic disorders and repeat offending: systematic review and meta-analysis. Schizophrenia Bulletin, 37(4), 800-810. doi:10.1093/schbul/sbp135

Felitti, V., Anda, R. F., Nordenberg, D. F., Williamson, D. F., Spitz, A. M., Edwards, V., ... Marks, J. S. (1998). Relationship of childhood abuse and household dysfunction to many of the leading causes of death in adults: the Adverse Childhood Experiences (ACE) Study. American Journal of Preventive Medicine 14, 245-258. doi:10.1016/S0749-3797(98)00017-8

Ferrando, S. J., \& Batki, S. L. (2000). Substance abuse and HIV infection. New Directions for Mental Health Services, 2000(87), 57-67. doi: 10.1002/yd.23320008709

Fichter, D. P. M., \& Quadflieg, D. P. N. (2005). Three-year course and outcome of mental illness in homeless men. European Archives of Psychiatry and Clinical Neuroscience, 255(2), 111120. doi: 10.1007/s00406-004-0543-9

Fisher, J., Mello, M. C. D., Patel, V., Rahman, A., Tran, T., Holton, S., \& Holmes, W. (2012). Prevalence and determinants of common perinatal mental disorders in women in low-and lower-middle-income countries: a systematic review. Bulletin of the World Health Organization, 90(2), 139-149. doi:10.2471/ BLT.11.091850

Foege, W. (1998). Adverse childhood experiences: a public health perspective. American Journal of Preventive Medicine 14 354-355.

Galván, F., Burnam, M., \& Bing, E., (2003). Co-occurring Psychiatric Symptoms and Drug Dependence or Heavy Drinking Among HIV-Positive People. Journal of Psychoactive Drugs, 35, 153160. doi: 10.1080/02791072.2003.10400510

Galván, J., \& Marín Navarrete, R. (2010). Sobre la atención a mujeres usuarias de drogas: cifras, barreras y modelo de tratamiento. En: Mendoza, M., Medina-Mora, M.E., \& Ajenjo, C.J.R. (Eds.). Mujeres y adicciones. (pp. 160-178). México: Instituto Nacional de Psiquiatría Ramón de la Fuente Muñiz. 
González, G., \& Rosenheck, R. A. (2002). Outcomes and service use among homeless persons with serious mental illness and substance abuse. Psychiatric Services, 53(4), 437-446. http:// dx.doi.org/10.1176/appi.ps.53.4.437

Grant, B. F., \& Dawson, D. A. (2006). Introduction to the national epidemiological survey on alcohol and related conditions. Alcohol Health \& Research World, 29(2), 74.

Grella, C. E. (1996). Background and overview of mental health and substance abuse treatment systems: Meeting the needs of women who are pregnant or parenting. Journal of Psychoactive Drugs, 28, 193-213.

Gum, A. M., King-Kallimanis, B., \& Kohn, R. (2009). Prevalence of mood, anxiety, and substance-abuse disorders for older Americans in the national comorbidity survey-replication. The American Journal of Geriatric Psychiatry, 17(9), 769-781. doi: http:// dx.doi.org/10.1097/JGP.0b013e3181ad4f5a

Gupta, M., Kumar, K., \& Garg, P. D. (2013). Dual diagnosis vs. triple diagnosis in HIV: A comparative study to evaluate the differences in psychopathology and suicidal risk in HIV positive male subjects. Asian Journal of Psychiatry, 6(6), 515-520. doi: http:// dx.doi.org/10.1016/j.ajp.2013.06.012

Hartwell, S., Deng, X., Fisher, W., Siegfriedt, J., Roy-Bujnowski, K., Johnson, C., \& Fulwiler, C. (2013). Predictors of accessing substance abuse services among individuals with mental disorders released from correctional custody. Journal of Dual Diagnosis, 9(1), 11-22. doi:10.1080/15504263.2012. 749449.

Hasin, D., Nunes, E., \& Meylan, J. (2004). Comorbidity of alcohol, drug and psychiatric disorders: epidemiology. En Kranzler, H., Tinsley, J., (Second Eth.). Dual Diagnosis and Psychiatric Treatment: Substance Abuse and Comorbid Disorders (pp. 1-34). USA: Marcel Bekker.

Hasin, D., \& Kilcoyne, B. (2012). Comorbidity of psychiatric and substance use disorders in the United States: current issues and findings from the NESARC. Current Opinion in Psychiatry, 25(3), 165. doi: 10.1097/YCO.0b013e3283523dcc.

Hawk, M., \& Davis, D. (2012). The effects of a harm reduction housing program on the viral loads of homeless individuals living with HIV/AIDS. AIDS care, 24(5), 577-582. doi: 10.1080/09540121.2011.630352

Houser, K. A., \& Welsh, W. (2014). Examining the association between co-occurring disorders and seriousness of misconduct

by female prison inmates. Criminal Justice and Behavior, 41(5), 650-666. doi: 10.1177/0093854814521195

Hwang, S. W., \& Burns, T. (2014). Health interventions for people who are homeless. The Lancet, 384(9953), 1541-1547. doi:10.1016/ S0140-6736(14)61133-8

Hwang, S. W. Tolomiczenko, G., Kouyoumdjian, F. G., \& Garner, R. E. (2005). Interventions to improve the health of the homeless: a systematic review. American Journal of Preventive Medicine, 29(4), 311-311. doi:10.1016/j.amepre.2005.06.017

Jiménez-Castro, L., Raventós-Vorst, H., \& Escamilla, M. (2011). Esquizofrenia y trastorno en el consumo de sustancias: prevalencia y características sociodemográficas en la población Latina. Actas Españolas de Psiquiatría, 39(2), 123-130.

Johnson, G., \& Chamberlain, C. (2008). Homelessness and substance abuse: Which comes first? Australian Social Work, 61(4), 342-356. doi: 10.1080/03124070802428191

Kerfoot, K. E., Petrakis, I. L., \& Rosenheck, R. A. (2011). Dual diagnosis in an aging population: Prevalence of psychiatric disorders, comorbid substance abuse, and mental health service utilization in the Department of Veterans Affairs. Journal of Dual Diagnosis, 7(1-2), 4-13. doi: 10.1080/15504263.2011.568306

Kessler, R. C., Berglund, P. A. Chiu, W. T., Demler, O., Heeringa, S., Hiripi, E., ... Zheng, H. (2004). The US national comorbidity survey replication (NCS-R): design and field procedures. International Journal of Methods in Psychiatric Research, 13(2), 69-92.

Kessler, R. C., Crum, R. M., Warner, L. A., Nelson, C. B., Schulenberg, J., \& Anthony, J. C. (1997). Lifetime co-occurrence of DSM-III-R alcohol abuse and dependence with other psychiatric disorders in the National Comorbidity Survey. Archives of General Psychiatry, 54(4), 313-321. doi:10.1001/archpsyc.1997.01830160031005.

King, V. L., Kidorf, M. S., Stoller, K. B., \& Brooner, R. K. (2000). Influence of psychiatric comorbidity on HIV risk behaviors: changes during drug abuse treatment. Journal of Addictive Diseases, 19(4), 65-83. doi: 10.1300/J069v19n04_07

Konstantakopoulos, G., Kakoulas, I., Valma, V., Stamatogiannopoulou, E., Giotakos, O., \& Katsadoros, K. (2003). Mental disorders and dual diagnosis in a sample of homeless people in Athens. Annals of General Hospital Psychiatry, 2(Suppl 1), 1-1.

Konstenius, M., Larsson, H., Lundholm, L., Philips, B., van de Glind, G., Jayaram-Lindström, N., \& Franck, J. (2015). An epidemiological study of ADHD, substance use, and comorbid problems in incarcerated women in Sweden. Journal of Attention Disorders, 19(1), 44-52. doi: 10.1177/1087054712451126

Kushel, M. B., Evans, J. L., Perry, S., Robertson, M. J., \& Moss, A. R. (2003). No door to lock: victimization among homeless and marginally housed persons. Archives of Internal Medicine, 163(20), 2492-2499. doi:10.1001/archinte.163.20.2492.

Lukasiewicz, M., Blecha, L., Falissard, B., Neveu, X., Benyamina, A., Reynaud, M., \& Gasquet, I. (2009). Dual diagnosis: prevalence, risk factors, and relationship with suicide risk in a nationwide sample of French prisoners. Alcoholism: Clinical and Experimental Research, 33(1), 160-168. doi: 10.1111/j.1530-0277.2008.00819.x

Mackesy-Amiti, M. E., Donenberg, G. R., \& Ouellet, L. J. (2012). Prevalence of psychiatric disorders among young injection drug users. Drug and Alcohol Dependence, 124(1), 70-78. doi:10.1016/j.drugalcdep.2011.12.012

Martin, M. S., Colman, I., Simpson, A. I., \& McKenzie, K. (2013). Mental health screening tools in correctional institutions: a sys- 
tematic review. BMC Psychiatry, 13(1), 275. doi:10.1186/1471$244 X-13-275$

Martínez-Lanz, P. M., \& Fernández Díaz, M. J. (2013). Domestic violence, alcohol consumption and depression in criminal population. Psychology, 4(03), 153. doi: http://dx.doi.org/10.4236/ psych.2013.43022

Martínez Lanz, P., Ocampo, D. B., Beyruti, L. R., \& de la Torre, A. B. (2012). Factores de riesgo asociados a la delincuencia masculina en México: un estudio a reclusos de un centro de readaptación social varonil. Criminalidad, 54(1), 359-377.

Mayston, R., Kinyanda, E., Chishinga, N., Prince, M., \& Patel, V. (2012). Mental disorder and the outcome of HIV/AIDS in low-income and middle-income countries: a systematic review. AIDS, 26, S117-S135. doi: 10.1097/QAD.0b013e32835bdeof

McCabe, B. E., Feaster, D. J., \& Mitrani, V. B. (2014). Health correlates of co-occurring substance use for women with HIV in cocaine use recovery. Addictive Behaviors, 39(3), 725-728. doi: http://dx.doi.org/10.1016/j.addbeh.2013.11.026

McHugo, G. J., Kammerer, N., Jackson, E. W., Markoff, L. S., Gatz, M., Larson, M. J., ... Hennigan, K. (2005). Women, co-occurring disorders, and violence study: Evaluation design and study population. Journal of Substance Abuse Treatment, 28(2), 91-107.

McKinnon, K., Cournos, F., Sugden, R., Guido, J. R., \& Herman, R. (1996). The relative contributions of psychiatric symptoms and AIDS knowledge to HIV risk behaviors among people with severe mental illness. The Journal of Clinical Psychiatry, 57(11), 506-513.

McKinnon, K. \& Rosner, J. (2000). Severe mental illness and HIV AIDS. New Directions for Mental Health Services, 2000(87), 6976. doi: 10.1002/yd.23320008710

McNiel, D. E., Binder, R. L., \& Robinson, J. C. (2005). Incarceration associated with homelessness, mental disorder, and co-occurring substance abuse. Psychiatric Services, 56(7), 840-846. http://dx.doi.org/10.1176/appi.ps.56.7.840

Medina-Mora, M.E. (2011). Introducción. En Mendoza, M., Medina-Mora, M.E., \& Ajenjo, C.J.R. (Eds.). Mujeres y adicciones. (pp. 13-18). México: Instituto Nacional de Psiquiatría Ramón de la Fuente Muñiz.

Mendoza-Meléndez, M. A., Borges-Guimaraes, G.L., Gallegos-Cari, A., Pacheco, J. Á. G., Hernández-Llanes, N. F., Camacho-Solís, R., \& Medina-Mora, M.E. (2015). Asociación del consumo de sustancias psicoactivas con el cuidado y la salud del adulto mayor. Salud Mental, 38, 15-26.

Miller, B. A., \& Downs, W. R. (1995). Violent victimization among women with alcohol problems. Recent Developments in Alcoholism, 12, 81- 101. doi: 10.1007/0-306-47138-8_5

Miquel, L., Roncero, C., García-García, G., Barral, C., Daigre, C., Grau-López, L., ... Casas, M. (2013). Gender differences in dually diagnosed outpatients. Substance Abuse, 34(1), 78-80. doi: 10.1080/08897077.2012.709223

Miquel, L., Roncero, C., Lopez-Ortiz, C., \& Casas, M. (2010). Epidemiological and diagnostic axis I gender differences in dual diagnosis patients. Adicciones, 23(2), 165-172.
Molnar, B. E., Buka, S. L., \& Kessler, R. C. (2001). Child sexual abuse and subsequent psychopathology: results from the $\mathrm{Na}$ tional Comorbidity Survey. American Journal of Public Health, 91(5), 753

Morse, S.A., MacMaster, S., Choi, S., \& Adams, S.M. (2015) Gender differences in treatment retention among individuals with co-occurring substance abuse and mental health disorders. Substance Use \& Misuse, 50(5), 653-663. doi 10.3109/10826084.2014.997828

Morse, S. A., Watson, C., MacMaster, S. A., \& Bride, B. E. (2015) Differences between older and younger adults in residential treatment for co-occurring disorders. Journal of Dual Diagnosis, 11(1), 75-82. doi: 10.1080/15504263.2014.993263

Montross, L. P., Barrio, C., Yamada, A. M., Lindamer, L., Golshan S., Garcia, P., ... Jeste, D. V. (2005). Tri-ethnic variations of co-morbid substance and alcohol use disorders in schizophrenia. Schizophrenia Research, 79(2), 297-305. doi:10.1016/j. schres.2005.04.014

Mundt, A. P., Alvarado, R., Fritsch, R., Poblete, C., Villagra, C., Kastner, S., \& Priebe, S. (2013). Prevalence rates of mental disorders in Chilean prisons. PloS One, 8, 1-8. doi:10.1371/journal. pone.0069109

Najavits, L.M., Weiss, R.D., \& Shaw, S.R. (1997) The link between substance abuse and posttraumatic stress disorder in women. A research review. The American Journal on Addictions, 6(4), 273-283. doi: 10.1111/j.1521-0391.1997.tb00408.x

Nelson, G., Stefancic, A., Rae, J., Townley, G., Tsemberis, S., Macnaughton, E., ... Goering, P. (2014). Early implementation evaluation of a multi-site housing first intervention for homeless people with mental illness: A mixed methods approach. Evaluation and Program Planning, 43, 16-26. doi:10.1016/j.evalprogplan.2013.10.004

Nielsen, S. F., Hjorthøj, C. R., Erlangsen, A., \& Nordentoft, M. (2011). Psychiatric disorders and mortality among people in homeless shelters in Denmark: a nationwide register-based cohort study. The Lancet, 377(9784), 2205-2214. doi:10.1016/S01406736(11)60747-2

Oslin, D., Liberto, J. G., O’Brien, J., Krois, S., \& Norbeck, J. (1997). Naltrexone as an adjunctive treatment for older patients with alcohol dependence. The American Journal of Geriatric Psychiatry, 5(4), 324-332.

Pascual, J. C., Malagón, A., Arcega, J. M., Gines, J. M., Navinés, R., Gurrea, A., ... Bulbena, A. (2008). Utilization of psychiatric emergency services by homeless persons in Spain. General Hospital Psychiatry, 30(1), 14-19. doi:10.1016/j.genhosppsych.2007.08.006

Patterson, M. L., Somers, J. M., \& Moniruzzaman, A. (2012). Prolonged and persistent homelessness: multivariable analyses in a cohort experiencing current homelessness and mental illness in Vancouver, British Columbia. Mental Health and Substance Use, 5(2), 85-101. doi: 10.1080/17523281.2011.618143 
Pickard, H., \& Fazel, S. (2013). Substance abuse as a risk factor for violence in mental illness: some implications for forensic psychiatric practice and clinical ethics. Current opinion in psychiatry, 26(4), 349. doi: 10.1097/YCO.0b013e328361e798

Platt, L., Grenfell, P., Fletcher, A., Sorhaindo, A., Jolley, E., Rhodes, T., \& Bonell, C. (2012). Systematic review examining differences in HIV, sexually transmitted infections and health-related harms between migrant and non-migrant female sex workers. Sexually Transmitted Infections, 89. 311-319. doi:10.1136/sextrans-2012-050491311

Polcin, D.L. (2015). Co-occurring substance abuse and mental health problems among homeless persons: suggestions for research and practice. Journal of Social Distress and the Homeless. In press.

Romero Mendoza, M. (2010). Mujeres y adicciones; reflexiones desde una perspectiva de género. En Romero Mendoza, M., Medina-Mora, M.E., \& Ajenjo, C.J.R. (Eds.). Mujeres y adicciones (pp. 19-36). México: Instituto Nacional de Psiquiatría Ramón de la Fuente Muñiz.

Romero Mendoza, M., Saldívar, G., Loyola, L., Rodríguez, E., \& Galván, J. (2010). Inequidades de género, abuso de sustancias y barreras al tratamiento en mujeres en prisión. Salud Mental, 33(6), 499-506.

Roncero C., Bachiller, D., Ros, E., \& Casas, M. (2011). Ancianos y drogodepencias. En: Bobes, J., Casas M., \& Gutiérrez, M. (Eds.), Manual de Trastornos Adictivos (2nd. Ed.). (pp. 268-275). Madrid: Enfoque Editorial S.C.,

Rosenberg, S. D., Drake, R. E., Brunette, M. F., Wolford, G. L., \& Marsh, B. J. (2005). Hepatitis C virus and HIV co-infection in people with severe mental illness and substance use disorders. Aids, 19, S26-S33.

Rosenberg, S. D., Goodman, L. A., Osher, F. C., Swartz, M. S., Essock, S. M., Butterfield, M. I.,... Salyers, M. P. (2001). Prevalence of HIV, hepatitis B, and hepatitis $\mathrm{C}$ in people with severe mental illness. American Journal of Public Health, 91(1), 31-37.

Ross, L. E., McLean, L. M., \& Psych, C. (2006). Anxiety disorders during pregnancy and the postpartum period: a systematic review. Depression, 6(9). 1-14. doi: 10.4088/JCP.v67n0818 .

Ruglass, L. M., Hien, D. A., Hu, M. C., \& Campbell, A. N. (2014). Associations between post-traumatic stress symptoms, stimulant use, and treatment outcomes: A secondary analysis of NIDA's women and trauma study. The American Journal on Addictions, 23(1), 90-95. doi: 10.1111/j.1521-0391.2013.12068.x

Sacco, P., Unick, G. J., Zanjani, F., \& Camlin, E. A. (2015). Hospital outcomes in major depression among older adults: differences by alcohol comorbidity. Journal of Dual Diagnosis, 11(1), 83-92. doi: 10.1080/15504263.2014.993295

Salmon, J. M., \& Forester, B. (2012). Substance abuse and co-occurring psychiatric disorders in older adults: A clinical case and review of the relevant literature. Journal of Dual Diagnosis, 8(1), 74-84. doi: 10.1080/15504263.2012.648439
Satre, D. D., Blow, F. C., Chi, F. W., \& Weisner, C. (2007). Gender differences in seven year alcohol and drug treatment outcomes among older adults. The American Journal on Addictions, 16(3), 216-221.

Satre, D. D., Mertens, J. R., Arean, P. A., \& Weisner, C. (2004). Five year alcohol and drug treatment outcomes of older adults versus middle-aged and younger adults in a managed care program. Addiction, 99(10), 1286-1297.

Schanzer, B., Dominguez, B., Shrout, P. E., \& Caton, C. L. (2007). Homelessness, health status, and health care use. American Journal of Public Health, 97(3), 464-469. doi: 10.2105/ AJPH.2005.076190

Shackman, J., \& Price, B. K. (2013). Mental health capacity building in northern Sierra Leone: lessons learned and issues raised. Intervention, 11(3), 261-275. doi: 10.1097/WTF.0000000000000010

Shaw, E., Levitt, C., Wong, S., \& Kaczorowski, J. (2006). Systematic review of the literature on postpartum care: effectiveness of postpartum support to improve maternal parenting, mental health, quality of life, and physical health. Birth, 33(3), 210-220. doi: 10.1111/j.1523-536X.2006.00106.x

Shaw, J., Senior, J., Lowthian, C., Foster, K., Clayton, R., Coxon, N., ... Hassan, L. (2009). A national evaluation of prison mental health in-reach services. Manchester: Offender Health Research Network, University of Manchester.

Shibre, T., Medhin, G., Alem, A., Kebede, D., Teferra, S., Jacobsson, L., ... Fekadu, A. (2015). Long-term clinical course and outcome of schizophrenia in rural Ethiopia: 10-year follow-up of a population-based cohort. Schizophrenia research, 161(2), 414-420. doi:10.1016/j.schres.2014.10.053

Sim, M., Hulse, G., \& Khong, E. (2004). Injecting drug use and skin. Australian Family Physician, 33(7), 1-4.

Steingrímsson, S., Carlsen, H. K., Sigfússon, S., \& Magnússon, A. (2012). The changing gender gap in substance use disorder: a total population based study of psychiatric in patients. Addiction, 107(11), 1957-1962. doi:10.1111/j.1360-0443.2012.03954.x

Stern, Y. (1994). Neuropsychological assessment of seropositive intravenous drug users. In I. Grant \& J. Martin (Eds.) The Neuropsychology of HIV Infection (pp.220-233) New York: Oxford University Press

Szerman, N., Martinez-Raga, J., Peris, L., Roncero, C., Basurte, I., Vega, P., ... Casas, M. (2013). Rethinking dual disorders/pathology. Addictive Disorders \& Their Treatment, 12(1), 1-10. doi: 10.1097/ADT.0b013e31826e7b6a

Torchalla, I., Strehlau, V., Li, K., \& Krausz, M. (2011). Substance use and predictors of substance dependence in homeless women. Drug and alcohol dependence, 118(2), 173-179. doi:10.1016/j. drugalcdep.2011.03.016

Topolovec-Vranic, J., Ennis, N., Colantonio, A., Cusimano, M. D., Hwang, S. W., Kontos, P., ... Stergiopoulos, V. (2012). Traumatic brain injury among people who are homeless: a systematic review. BMC Public Health, 12(1), 1059. doi:10.1186/1471-245812-1059 
Tsemberis, S., Gulcur, L., \& Nakae, M. (2004). Housing first, consumer choice, and harm reduction for homeless individuals with a dual diagnosis. American Journal of Public Health, 94(4), 651656.

Unger, J. B., Kipke, M. D., Simon, T. R., Montgomery, S. B., \& Johnson, C. J. (1997). Homeless youths and young adults in Los Angeles: Prevalence of mental health problems and the relationship between mental health and substance abuse disorders. American Journal of Community Psychology, 25(3), 371-394. doi:10.1023/A:1024680727864

Vet, R. de, van Luijtelaar, M. J., Brilleslijper-Kater, S. N., Vanderplasschen, W., Beijersbergen, M. D., \& Wolf, J. R. (2013). Effectiveness of case management for homeless persons: a systematic review. American Journal of Public Health, 103(10), e13-e26. doi: 10.2105/AJPH.2013.301491

Villagrá, P., Fernández, P., García-Vega, E., \& González-Menéndez, A. (2013). Dual diagnosis in prisoners: Childhood sexual and physical abuse as predictors in men and women. International Journal of Offender Therapy and Comparative Criminology, In press. doi: 10.1177/0306624X13513560

Weiss, M. J. S., \& Wagner, S. H. (1998). What explains the negative consequences of adverse childhood experiences on adult health. American Journal of Preventive Medicine, 14(4), 356-360.

White, E. F., Garfein, R. S., Brouwer, K. C., Lozada, R., Ramos, R., Firestone-Cruz, M., ... Strathdee, S. A. (2007). Prevalence of hepatitis $\mathrm{C}$ virus and HIV infection among injection drug users in two Mexican cities bordering the US. Salud Pública de México, 49(3), 165-172.
Whetten, K., Reif, S., Swartz, M., Stevens, R., Ostermann, J. Hanisch, L., \& Eron Jr, J. J. (2005). A brief mental health and substance abuse screener for persons with HIV. AIDS Patient Care \& STDs, 19(2), 89-99.

Wood, S. R., \& Buttaro, A. (2013). Co-occurring severe mental illnesses and substance abuse disorders as predictors of state prison inmate assaults. Crime \& Delinquency, 59(4), 510-535. doi: $10.1177 / 0011128712470318$

World Health Organization (WHO). (2010). Atlas on substance use (2010): resources for the prevention and treatment of substance use disorders. Geneva: World Health Organization. Recuperado de http://apps.who.int/iris/handle/10665/44455

Yovtcheva, S. P., Rifai, M. A., Moles, J. K., \& Van Der Linden, B. J. (2001). Psychiatric comorbidity among hepatitis C-positive patients. Psychosomatics, 42(5), 411-415. doi:10.1176/Appi. psy.42.5.411

Zilberman, M. L., Tavares, H., Blume, S. B., \& Guebaly, N. (2003). Substance use disorders: sex differences and psychiatric comorbidities. Canadian Journal of Psychiatry, 48(1), 5-13.

Zlotnick, C., Clarke, J.G., Friedmann, P.D., Roberts, M.B., Sacks, S., \& Melnick, G. (2008). Gender differences in comorbid disorders among offenders in prison substance abuse treatment programs. Behavioral Sciences \& the Law, 26(4): 403-412. doi: 10.1002/bsl.831 